\title{
The effect of using nefopam in fentanyl-based intravenous patient-controlled analgesia on the incidence of postoperative nausea and vomiting in laparoscopic gynecological surgery
}

\author{
YoonJu Go, Jun-Young Chung, Jae-woo Yi, Bong-Jae Lee, Hyungseok Seo \\ Department of Anesthesiology and Pain Medicine, Kyung Hee University Hospital at Gangdong, College of Medicine, \\ Kyung Hee University, Seoul, Korea
}

Received December 1, 2020

Revised December 11, 2020

Accepted December 23, 2020

Corresponding author

Hyungseok Seo

Department of Anesthesiology

and Pain Medicine, Kyung Hee

University Hospital at Gangdong,

892 Dongnam-ro, Gangdong-gu,

Seoul 05278, Korea

Tel: +82-2-440-7808

Fax: +82-2-440-7806

E-mail: seohyungseok@gmail.com ORCID:

http://orcid.org/0000-0003-4574-9122
Currently, the regimen of intravenous patient-controlled analgesia (IV-PCA) has become lowering opioid to reduce opioid-related side effects, particularly, postoperative nausea and vomiting (PONV). Using nefopam in IV-PCA may decrease the incidence of PONV, but there is still a lack of evidence. 178 patients undergoing laparoscopic gynecological surgery were randomly assigned into two groups. The IV-PCA regimen for group F was $20 \mu \mathrm{g} / \mathrm{kg}$ fentanyl and for group N was $10 \mu \mathrm{g} / \mathrm{kg}$ fentanyl with $200 \mathrm{mg}$ of nefopam. The severity of PONV and pain were assessed at both 1 hour and 24 hours after surgery. Patients' satisfaction with recovery was evaluated by a structured questionnaire. The incidence of PONV showed no statistical difference between group $\mathrm{N}$ and group $\mathrm{F}(28.2 \%$ vs. $26.8 \%$ at 1 hour, $\mathrm{p}=0.839$ and $32.1 \%$ vs. $43.9 \%$ at 24 hours, $p=0.120$, respectively). Also, postoperative pain intensity showed no statistical difference between the two groups. Patient recovery was similar between the two groups. The combined use of nefopam in IV-PCA seems to effectively decrease the fentanyl dose in laparoscopic gynecological surgery. However, it did not contribute to decreasing the incidence of PONV.

Keywords: Patient-controlled analgesia; Fentanyl; Postoperative nausea and vomiting; Nefopam

\section{INTRODUCTION}

Postoperative nausea and vomiting (PONV) has many risk factors, including female gender, non-smoker, previous history of PONV, and opioids use after operation [1]. Preoperatively, the risk of PONV can be assessed by patient-related factors [2-5]. General anesthesia, particularly volatile anesthetics or duration of anesthesia showed positive association with PONV [1,6]. Although the type of surgery is still debating, laparoscopic gynecological surgery or cholecys- tectomy independently increase the risk of PONV [1,4,7,8].

Opioids can provide optimal postoperative analgesia and patient satisfaction especially in major surgery [9,10], but also cause PONV in approximately one-third of patients [11]. Considering the adverse effect of opioid such as PONV, current intravenous patient-controlled analgesia (IV-PCA) regimen has become lowering opioid amount and using non-opioid analgesics, such as nefopam. Although nefopam showed fentanyl-sparing effect [12], in patients of higher PONV risk, the effect of using nefopam in IV-PCA is 
still lack of evidence for decreasing the incidence of PONV. In the present study, we added nefopam in IV-PCA and compared the incidence of PONV in patient with moderate to severe PONV risk.

\section{MATERIALS AND METHODS}

\section{Patients}

We enrolled female patients who were scheduled to undergo laparoscopic gynecological surgery between 19 and 75 years of age with American Society of Anesthesiologists (ASA) physical status classification I-III. Exclusion criteria was body mass index (BMI) $<18.5 \mathrm{~kg} / \mathrm{m}^{2}$ or $>35.0 \mathrm{~kg} / \mathrm{m}^{2}$, current or ex-smokers, uncontrolled diabetic mellitus (recent 3-month hemoglobin $\mathrm{A} 1 \mathrm{c} \geq 6.5 \%$ ) and patients with severe comorbidity who need to reduce the amount of fentanyl. According to Apfel score [2], patients are expected to have moderate to severe PONV risk (Apfel score 3 or 4). All patients received instruction before the day of surgery about the study protocol and the 10-point numeric rating scale (NRS) for nausea and pain ( $0=$ nausea or pain-free and $10=$ the most severe nausea or pain ever). A written informed consent was obtained from all the participants. This investigation was approved by the Kyung Hee University Hospital at Gangdong Institutional Review Board (IRB\#: KHNMC 2018-10-018-001) and registered at national clinical research registry (KCT0003659, http://cris.nih.go.kr) prior to patient enrollment.

\section{Randomization, allocation, and blindness}

The enrolled patients were randomly assigned into one of two groups, group $\mathrm{N}$ for using nefopam in IV-PCA and group F for fentanyl-only in IV-PCA. For randomization, a computer-generated, the random 4 and 6 blocks technique was used. An independent investigator kept the random assignment table for participants before surgery, and noticed to research team, of the patient allocation, on the day of the surgery.

The IV-PCA regimen for group $\mathrm{N}$ was $10 \mu \mathrm{g} / \mathrm{kg}$ fentanyl with $200 \mathrm{mg}$ of nefopam and for group F was $20 \mu \mathrm{g} / \mathrm{kg}$ fentanyl. Ramosetron $0.6 \mathrm{mg}$ was added and remained volume was replaced with $0.9 \% \mathrm{NaCl}$ to make total volume $100 \mathrm{~mL}$ in the both regimens. The IV-PCA machine (AutoMed3200 ${ }^{\circledR}$, Ace Medical, Seoul, Korea) was set to basal infusion rate of 1
$\mathrm{mL} /$ hour and patient-controlled bolus injection volume of 1 $\mathrm{mL}$ with 15 minutes of the lock-out interval time. The maximal infusion volume was $5 \mathrm{~mL}$ per hour. For maintaining blindness, the dose prescription label in IV-PCA machine of both groups was marked as the study drug.

\section{Procedures}

On arrival in the operating room, standard monitoring including electrocardiogram, peripheral oxygen saturation, end-tidal carbon dioxide concentration, bispectral index, and non-invasive blood pressure monitoring was applied. Then glycopyrrolate $0.2 \mathrm{mg}$ and dexamethasone $5 \mathrm{mg}$ were given before anesthesia induction. After preoxygenation with 100\% oxygen for 5 minutes, anesthesia was induced and maintained with target controlled infusion of propofol (Schnider model, Ce: $4.0 \mu \mathrm{g} / \mathrm{mL}$ ) and remifentanil (Minto model, Ce: $4.0 \mathrm{ng} / \mathrm{mL}$ ) using the Orchestra ${ }^{\circledR}$ Base Prima target-controlled infusion system (Fresenius Kabi, Bad Homburg, Germany). Anesthesia depth was maintained using bispectral index between 40-60 during surgery. After loss of consciousness, rocuronium bromide $0.6 \mathrm{mg} / \mathrm{kg}$ was given and airway was maintained with tracheal tube and the lungs were ventilated with a mix of oxygen and medical air. Tidal volume $6-8 \mathrm{~mL} / \mathrm{kg}$ and respiratory rate $10-16 /$ minute was set to maintain end-tidal carbon dioxide concentration between 35 and $40 \mathrm{mmHg}$. At the end of surgery, neuromuscular blockade was reversed using sugammadex or mixture of glycopyrrolate and neostigmine.

In both groups, $20 \mathrm{mg}$ of nefopam was given over 10 minutes when skin closure begins. For maintaining double-blindness, IV-PCA was prepared and connected to the patient by an independent physician who did not participated with the study.

In the post-anesthesia care unit (PACU), PONV assessed with NRS was assessed at 1 hour after admission. Patients experiencing PONV score $\geq 4$ was given $4 \mathrm{mg}$ of ondansetron for the first rescue drug. The second rescue drug, $10 \mathrm{mg}$ metoclopramide was slowly infused intravenously if PONV score was still $\geq 4$ at 20 minutes after the first rescue drug administration. Pain score assessed with NRS at the time of PACU admission and 1 hour later. If pain score was $<6$ and patient wanted pain control, $1 \mathrm{~g}$ of intravenous acetaminophen was given. If pain score $\geq 7$, fentanyl $0.5 \mu \mathrm{g} / \mathrm{kg}$ was given intravenously. If pain score was not relieved after 
20 minutes, the same amount of fentanyl was administered repeatedly.

\section{Outcome measurement}

The primary outcomes were PONV score at 1 hour and 24 hours after surgery with the number of vomiting events during each time interval. Secondary endpoints in this study were pain score at 1 and 24 hours after surgery, total amount and kinds of analgesics and antiemetics given in PACU and at ward, the amount of IV-PCA consumption during postoperative 24 hours, and patient satisfaction evaluated by Quality of Recovery 15 (QoR-15) questionnaire [13] at 24 hours after surgery.

\section{Sample size calculation and statistical analysis}

Sample size was estimated on the basis of the previous study by Apfel et al. [2] which reported approximately $40 \%$ of PONV incidence with patient who has more than 2 PONV risk factors. Assuming lowering fentanyl dose in IV-
PCA regimen reduces PONV incidence by $50 \%$ than fentanyl-only regimen, 81 patients per group would be required for an experimental design, using a type 1 error of $5 \%$ and desired power of $80 \%$. With a possibility of $10 \%$ loss due to any unexpected circumstances, we elected to recruit 89 patients per group into the study.

All patients were analyzed on an intention-to-treat analysis. If there was any critical missing data or drop-out of any participant, the reason of exclusion was clarified and summarized in the clinical study report. Continuous data were analyzed by the Student t-test or the Mann-Whitney $\mathrm{U}$ test depending on the presence of normality. Categorical data were analyzed using Chi-square analysis or Fisher's exact test where applicable. Statistical analysis was performed using a standard statistical program (Medcalc, Version 18.11.3). All values are expressed as a mean \pm standard deviation, median [quartiles] (minimum, maximum), or number (percentage).

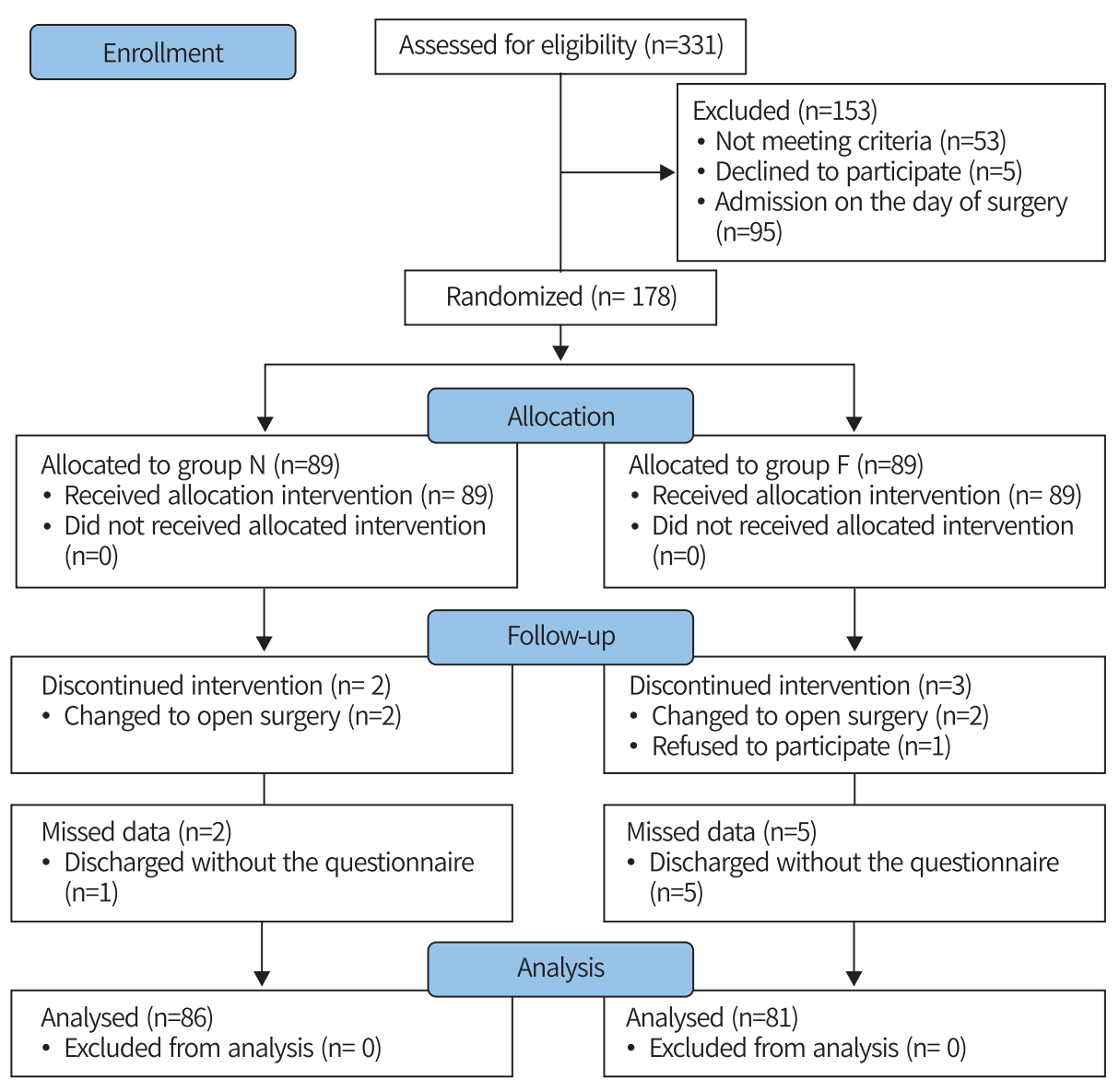

Fig. 1. A CONSORT flow diagram. 


\section{RESULTS}

In a total of 178 enrolled patients, 5 patients were droppedout and the data of 6 patients was missed. Finally, 167 vpatients were analyzed (Fig. 1). Patients' demographics are shown in Table 1. No significant difference was observed between two groups. Intraoperative data were similar between the two groups (Table 1).

The overall incidence of PONV within postoperative 24 hours was $38.0 \%$. The incidence of PONV showed no statistical difference between group $\mathrm{N}$ and group $\mathrm{F}(28.2 \%$ vs.

Table 1. Patients' demographics and intraoperative data

\begin{tabular}{lccc}
\hline \multicolumn{1}{c}{ Variables } & Group N $(\mathrm{n}=86)$ & Group $\mathrm{F}(\mathrm{n}=81)$ & $\mathrm{p}$ value \\
\hline Patient demographics & & & \\
Age, yrs & $44 \pm 10$ & $43 \pm 11$ & 0.406 \\
Height, $\mathrm{cm}$ & $159 \pm 6$ & $159 \pm 6$ & 0.755 \\
Weight, $\mathrm{kg}$ & $61 \pm 11$ & $60 \pm 10$ & 0.712 \\
BMI, kg/cm & $23.6 \pm 4.1$ & $23.2 \pm 3.4$ & 0.976 \\
ASA PS I/II/III & $70 / 13 / 2$ & $62 / 18 / 2$ & 0.354 \\
& $(82.4 / 15.3 / 2.4 \%)$ & $(75.6 / 22.0 / 2.4 \%)$ & \\
Intraoperative data & & & \\
Operation time, min & $83 \pm 39$ & $92 \pm 46$ & 0.175 \\
Anesthesia duration, min & $118 \pm 41$ & $126 \pm 49$ & 0.207 \\
Propofol amount, mg & $802 \pm 278$ & $865 \pm 434$ & 0.258 \\
Remifentanil amount, $\mathrm{mg}$ & $1,677 \pm 762$ & $1,918 \pm 1,001$ & 0.081 \\
Intraoperative fluid input, & $602 \pm 279$ & $701 \pm 469$ & 0.095 \\
$\quad$ mL & & & \\
Urine output, mL & $82 \pm 66$ & $108 \pm 135$ & 0.506 \\
Estimated blood loss, $\mathrm{mL}$ & $80 \pm 129$ & $93 \pm 172$ & 0.980 \\
\hline
\end{tabular}

Data presented as mean \pm standard deviation or number (\%). BMl: body mass index, ASA PS: American Society of Anesthesiologists physical status.

Table 2. Incidence of postoperative nausea and vomiting and antiemetics

\begin{tabular}{lccc}
\hline \multicolumn{1}{c}{ Variables } & Group N (n=86) & Group F (n=81) & p value \\
At postoperative 1 hour & & & \\
Nausea & $24(28.2)$ & $22(26.8)$ & 0.839 \\
Nausea_NRS & $0[0-1](0,9)$ & $0[0-1](0,6)$ & 0.542 \\
Vomiting & 0 & 0 & \\
Antiemetics & $11(12.9)$ & $3(3.6)$ & 0.071 \\
Until postoperative 24 hours & & & \\
Nausea & $27(32.1)$ & $36(43.9)$ & 0.120 \\
Nausea_NRS & $0[0-1](0,8)$ & $0[0-1](0,8)$ & 0.459 \\
Vomiting & $4(4.7)$ & $9(11.0)$ & 0.132 \\
Antiemetics & $5(5.9)$ & $13(15.8)$ & 0.026
\end{tabular}

Data presented as nunber (\%) or median [quartiles] (minimum, maximum). NRS: numerical rating scale.
$26.8 \%$ at 1 hour, $\mathrm{p}=0.839$ and $32.1 \%$ vs. $43.9 \%$ at 24 hours, $\mathrm{p}=0.120$, respectively). The severity of nausea and the incidence of rescue antiemetic use were similar between two groups (Table 2).

Postoperative pain was no significant difference between group $\mathrm{N}$ and $\mathrm{F}$ (Table 3). The incidence of rescue analgesics (number needed to treat=18.2), total infused IV-PCA amount, the discontinuation rate of PCA before 24 hours, and the quality of recovery showed no significant difference between the two groups (Table 3).

\section{DISCUSSION}

In the present study, IV-PCA regimen with using nefopam did not reduce PONV incidence compared to conventional fentanyl-based regimen in patients with moderate to severe PONV risk. However, the two IV-PCA regimens showed similar effect on postoperative pain.

Regardless of several undesirable adverse effects, opioid based IV-PCA has been widely used for postoperative analgesia in patients with moderate to severe pain [10]. However, it also can be a major cause of PONV [1]. Growing body of literatures have explored to reduce opioid amount by co-administration of drugs such as non-steroidal anti-inflammatory drugs, paracetamol and ketamine [14,15].

Table 3. Postoperative outcomes

\begin{tabular}{|c|c|c|c|}
\hline Variables & $\begin{array}{c}\text { Group N } \\
(n=86)\end{array}$ & $\begin{array}{c}\text { Group F } \\
(n=81)\end{array}$ & $p$ value \\
\hline \multicolumn{4}{|l|}{ At postoperative 1 hour } \\
\hline Pain_VAS & $5[3-6]$ & $5[4-6]$ & 0.252 \\
\hline \multirow[t]{2}{*}{ Rescue analgesics } & 71 (83.5) & $73(89.0)$ & 0.117 \\
\hline & & & $(\mathrm{NNT}=18.2)$ \\
\hline Rescue opioid amount, mg & 50 [30-70] & 55 [30-90] & 0.240 \\
\hline \multicolumn{4}{|l|}{ Until postoperative 24 hours } \\
\hline Pain_VAS & $4[2-5]$ & $3[2-5]$ & 0.428 \\
\hline \multirow[t]{2}{*}{ Rescue analgesics } & $28(32.9)$ & $28(34.1)$ & 0.869 \\
\hline & & & $(\mathrm{NNT}=83.3)$ \\
\hline Rescue opioid amount, mg & $50[0-75]$ & $50[25-75]$ & 0.978 \\
\hline QoR-15 & 104 [84-120] & 94 [78-110] & 0.196 \\
\hline Total infused PCA, mL & $\begin{array}{c}28.9 \\
{[26.3-33.1]}\end{array}$ & $\begin{array}{c}29.1 \\
{[28.3-33.8]}\end{array}$ & 0.487 \\
\hline PCA discontinuation & $5(5.9)$ & $7(8.5)$ & 0.508 \\
\hline
\end{tabular}

Data presented as number (\%) or median [quartiles]. NNT: number needed to treat, QoR-15: quality of recovery 15. 
Nefopam is a non-opioid, non-steroidal, centrally acting analgesics and does not bind to opioid receptors [16-19], which shows different inherent analgesic mechanism from opioids. Because of nefopam has been reported to be 20$50 \%$ of opioids-sparing effect [20-22], using nefopam to IV-PCA may decrease PONV incidence by reducing opioid amount.

Our result revealed that the using nefopam in IV-PCA did not reduce PONV incidence, compared to the conventional fentanyl-based regimen in the patient with moderate to severe PONV risk. It may suggest that reducing opioid amount in IV-PCA solely is not sufficient to decrease PONV, because PONV is affected by various factors such as sex, laparoscopic surgery, pain, etc. [1]. Also, to control breakthrough pain, non-opioid analgesics other than opioids seemed to be less effective, thereby opioids being inevitably needed. Moreover, strictly followed the consensus PONV guideline [5] may contribute to lower the overall PONV incidence, and diminish the inter-group difference. Effective PONV prophylaxis needs multidisciplinary approach such as adequate hydration, using dexamethasone, and propofol for anesthesia maintenance, etc., which were all included in this study protocol [5]. Due to our multidisciplinary prophylaxis, the overall incidence of PONV was only 38\%, despite of known incidence of $60 \%$ in patients with the similar risk [2].

Considering the balance between analgesic effect and adverse effects of opioid, proper use of nefopam to reduce opioid amount would be a critical issue. Balanced combination of analgesics with different mechanism can effectively decrease pain intensity without adverse effects of individual drugs. However, excessive amount of total analgesics could exert or deepen the side effect of each drugs even though they do not share analgesic mechanism. Without dose reduction of fentanyl, additional use of nefopam increased PONV incidence [12]. It may suggest that, for an appropriate opioid sparing effect of nefopam, the deliberately calculated dose would be necessary. In our result, we added nefopam under calculation considering fentanyl-equivalent dose. Consequently, the regimen using nefopam in IV-PCA provided an effective postoperative analgesia with minimized side effects, compared to fentanyl-only regimen. Therefore, using nefopam in IV-PCA can be a reasonable option for the conventional fentanyl-based regimen

The balance of nefopam effects between the incidence of
PONV and postoperative pain has been controversial. Although previous reports compared the analgesic or adverse effect between nefopam and opioids, each study showed an incoherency in their methods, particularly in the study population, anesthetic setting, the type of surgery, etc. [23]. Merle et al. suggested that in the less painful surgery, the analgesic effect of nefopam could be limited, and even cause adverse effect [24]. In Choi et al. with maxillofacial surgery, nefopam did not reduce PONV incidence compared with fentanyl, despite of similar analgesic effect [25]. However, several studies showed that nefopam was effective for moderate to high postoperative pain control [26-29]. In our result, as is consistent with previous results, using nefopam in IV-PCA showed similar effectiveness to conventional fentanyl-based regimen.

There are several limitations in the present study. First, we followed up patients only twice during the entire study period. Because nausea is a subjective symptom, it could be insufficient to investigate with single or short-term follow-up. Also, until postoperative 24 hours, the patient was not be fully recovered, thereby may not provide accurate information. Second, because our data at postoperative 24 hours was mainly based on nursing report in the electronic medical record system, those may have inter-individual differences or increased risk of human errors. For example, some nurse recorded patient information in detail, but others even did not remember precisely or omit recording. Third, in contrast to the PACU, the patient evaluation can be less controlled and be incoherent in general ward. In PACU, single investigator consistently followed the patients, and the study protocol can be well controlled. However, in the ward, nursing staffs generally rotate, thereby the study protocol cannot be strictly followed up.

In conclusion, although its similar analgesic effect, using nefopam as an adjuvant to opioids in IV-PCA did not reduce the incidence of PONV in laparoscopic gynecological surgery.

\section{ACKNOWLEDGEMENTS}

The present study is a doctoral dissertation which was submitted at the Kyung Hee University, Graduate School, Department of Medicine. 


\section{CONFLICT OF INTEREST}

No potential conflict of interest relevant to this article was reported.

\section{REFERENCES}

1. Apfel CC, Heidrich FM, Jukar-Rao S, Jalota L, Hornuss C, Whelan RP, et al. Evidence-based analysis of risk factors for postoperative nausea and vomiting. Br J Anaesth 2012;109: 742-53.

2. Apfel CC, Läärä E, Koivuranta M, Greim CA, Roewer N. A simplified risk score for predicting postoperative nausea and vomiting: conclusions from cross-validations between two centers. Anesthesiology 1999;91:693-700.

3. Eberhart LH, Högel J, Seeling W, Staack AM, Geldner G, Georgieff M. Evaluation of three risk scores to predict postoperative nausea and vomiting. Acta Anaesthesiol Scand 2000;44:480-8.

4. Stadler M, Bardiau F, Seidel L, Albert A, Boogaerts JG. Difference in risk factors for postoperative nausea and vomiting. Anesthesiology 2003;98:46-52.

5. Gan TJ, Diemunsch P, Habib AS, Kovac A, Kranke P, Meyer $\mathrm{TA}$, et al. Consensus guidelines for the management of postoperative nausea and vomiting. Anesth Analg 2014;118:85113.

6. Apfel CC, Philip BK, Cakmakkaya OS, Shilling A, Shi YY, Leslie JB, et al. Who is at risk for postdischarge nausea and vomiting after ambulatory surgery? Anesthesiology 2012;117:47586.

7. Cohen MM, Duncan PG, DeBoer DP, Tweed WA. The postoperative interview: assessing risk factors for nausea and vomiting. Anesth Analg 1994;78:7-16.

8. Apfel CC, Kranke P, Eberhart LH. Comparison of surgical site and patient's history with a simplified risk score for the prediction of postoperative nausea and vomiting. Anaesthesia 2004;59:1078-82.

9. Hudcova J, McNicol E, Quah C, Lau J, Carr DB. Patient controlled opioid analgesia versus conventional opioid analgesia for postoperative pain. Cochrane Database Syst Rev 2006; (4):CD003348.

10. Gritsenko K, Khelemsky Y, Kaye AD, Vadivelu N, Urman RD. Multimodal therapy in perioperative analgesia. Best Pract Res Clin Anaesthesiol 2014;28:59-79.

11. Culebras X, Corpataux JB, Gaggero G, Tramèr MR. The antiemetic efficacy of droperidol added to morphine patientcontrolled analgesia: a randomized, controlled, multicenter dose-finding study. Anesth Analg 2003;97:816-21.

12. Jin HS, Kim YC, Yoo Y, Lee C, Cho CW, Kim WJ. Opioid sparing effect and safety of nefopam in patient controlled analgesia after laparotomy: a randomized, double blind study. J Int Med Res 2016;44:844-54.

13. Stark PA, Myles PS, Burke JA. Development and psychometric evaluation of a postoperative quality of recovery score: the QoR-15. Anesthesiology 2013;118:1332-40.

14. Hyllested M, Jones S, Pedersen JL, Kehlet H. Comparative effect of paracetamol, NSAIDs or their combination in postoperative pain management: a qualitative review. Br J Anaesth 2002;88:199-214.

15. Wang L, Johnston B, Kaushal A, Cheng D, Zhu F, Martin J. Ketamine added to morphine or hydromorphone patientcontrolled analgesia for acute postoperative pain in adults: a systematic review and meta-analysis of randomized trials. Can J Anaesth 2016;63:311-25.

16. Heel RC, Brogden RN, Pakes GE, Speight TM, Avery GS. Nefopam: a review of its pharmacological properties and therapeutic efficacy. Drugs 1980;19:249-67.

17. Kim KH, Abdi S. Rediscovery of nefopam for the treatment of neuropathic pain. Korean J Pain 2014;27:103-11.

18. Piercey MF, Schroeder LA. Spinal and supraspinal sites for morphine and nefopam analgesia in the mouse. Eur J Pharmacol 1981;74:135-40.

19. Conway AC, Mitchell CL. Analgesic studies with nefopam hydrochloride. Arch Int Pharmacodyn Ther 1977;226:156-71.

20. Mimoz O, Incagnoli P, Josse C, Gillon MC, Kuhlman L, Mirand A, et al. Analgesic efficacy and safety of nefopam vs. propacetamol following hepatic resection. Anaesthesia 2001;56:520-5.

21. Du Manoir B, Aubrun F, Langlois M, Le Guern ME, Alquier C, Chauvin M, et al. Randomized prospective study of the analgesic effect of nefopam after orthopaedic surgery. Br J Anaesth 2003;91:836-41.

22. McLintock TT, Kenny GN, Howie JC, McArdle CS, Lawrie S, Aitken $\mathrm{H}$. Assessment of the analgesic efficacy of nefopam hydrochloride after upper abdominal surgery: a study using patient controlled analgesia. Br J Surg 1988;75:779-81.

23. Park SK, Yoo S, Kim WH, Lim YJ, Bahk JH, Kim JT. Association of nefopam use with postoperative nausea and vomiting in gynecological patients receiving prophylactic ramosetron: a retrospective study. PLoS One 2018;13:e0199930.

24. Merle JC, Vandroux D, Odin I, Dupuis JL, Bougault A, Mehaddi $Y$, et al. [Analgesic effect of continuous intravenous nefopam after urological surgery]. Ann Fr Anesth Reanim 2005;24:13-8. French.

25. Choi E, Karm MH, So E, Choi YJ, Park S, Oh Y, et al. Effects on postoperative nausea and vomiting of nefopam versus fentanyl following bimaxillary orthognathic surgery: a prospective double-blind randomized controlled trial. J Dent Anesth Pain Med 2019;19:55-66. 
26. Moon JY, Choi SS, Lee SY, Lee MK, Kim JE, Lee JE, et al. The effect of nefopam on postoperative fentanyl consumption: a randomized, double-blind study. Korean J Pain 2016;29:1108.

27. Oh CS, Jung E, Lee SJ, Kim SH. Effect of nefopam- versus fentanyl-based patient-controlled analgesia on postoperative nausea and vomiting in patients undergoing gynecological laparoscopic surgery: a prospective double-blind randomized controlled trial. Curr Med Res Opin 2015;31:1599-607.
28. Kim SY, Huh KH, Roh YH, Oh YJ, Park J, Choi YS. Nefopam as an adjunct to intravenous patient-controlled analgesia after renal transplantation: a randomised trial. Acta Anaesthesiol Scand 2015;59:1068-75.

29. Kim K, Kim WJ, Choi DK, Lee YK, Choi IC, Sim JY. The analgesic efficacy and safety of nefopam in patient-controlled analgesia after cardiac surgery: a randomized, double-blind, prospective study. J Int Med Res 2014;42:684-92. 